\title{
Critical Investigation of the Combined Compliance Average and Spreading Measures in the Robust Topology Optimization with Uncertain Loading Magnitude and Direction
}

\author{
Anikó Csébfalvi ${ }^{1 *}$ \\ ${ }^{1}$ Department of Civil Engineering, University of Pécs, 2 Boszorkány, Pécs, H-7634, Hungary \\ ${ }^{*}$ Corresponding author, e-mail: csebfalvi@mik.pte.hu
}

Received: 16 June 2020, Accepted: 27 August 2020, Published online: 29 September 2020

\begin{abstract}
The paper critically investigates the role of the combined compliance average and spreading measures in the volume-constrained continuous robust topology optimization with uncertain loading magnitude and direction. In the robust topology optimization the generally expected and most popular robustness measure is the expected compliance, In the expectancy oriented approach, the compliance increment which is needed to get the robust design is an implicitly defined response variable. In order to open the possibility of the creative contribution of the designer to the best robust design searching process, this measure is sometimes combined with a spreading-oriented measure, which may be the variance or standard deviation. The best weighting schema can be done by a try-and-error-like algorithm in which the weights are design variables and the compliance-increment remains an implicitly defined response variable. In this paper, it will be shown that all of the compliance oriented approaches which are based on a single or combined statistical measure can be replaced by a new compliance-function-shape-oriented robust approach in which the allowedcompliance-increment will be an explicitly defined design variable and for a given increment value the robust solution will be the theoretically best one. A popular volume-constrained symmetric bridge problem with uncertain loading magnitude and direction will be presented to demonstrate the viability and efficiency of the proposed robust approach.
\end{abstract}

Keywords

topology optimization, uncertain loads, robust optimization, expected compliance, compliance spreading, total compliance variation

\section{Introduction}

Uncertainty is an important consideration in volume-constrained continuous topology optimization to produce robust solutions which are insensitive to the uncertain design parameters as much as possible. More than two decades ago a robust truss topology design procedure was elaborated by Ben-Tal and Nemirovski [1] using semidefinite programming as one of the first application. The source of uncertainty may be the variability of applied loads, spatial positions of nodes, material properties, and so on [2-5]. Various deterministic and stochastic approaches have been developed to account for different types of uncertainty in structural design and optimization methods to get robust solutions [6-8]. In addition, the reliability based topology optimization play important rule to handle uncertainties [9-13] in this research field.

In this paper, it is assumed that the investigated problems are symmetric, the only source of uncertainty is the variabi- lity of the magnitude and direction of the loads and a compliance-based performance measure is used in the robust topology optimization to get a robust design. A good summary of the different approaches of the compliance-based robust topology optimization can be found in [14].

In the volume-constrained continuous robust topology optimization with uncertain loading magnitude and direction the generally expected and most popular robustness measure is the expected compliance. In the expectancy oriented approach, the compliance increment which is needed to get the robust design is an implicitly defined response variable. In order to open the possibility of the creative contribution of the designer to the best robust design searching process, this measure is sometimes combined with a spreading-oriented measure, which may be the variance or standard deviation [14-20]. In these cases, the best weighting schema can be done by a try-and-error-like 
algorithm in which the weights are design variables and the compliance-increment remains invariably an implicitly defined response variable.

In the presented case, according to the symmetricity assumption, the robust nominal compliance is always greater than or equal to the original nominal compliance and the robust compliance function shape is always more balanced than the nominal compliance function shape on the set of uncertain design parameters independently from the applied robustness measure. In a forthcoming paper, an anomaly resolving strategy will be presented for such challenging and frustrating cases where, independently from the applied robust approach, the original nominal and robust nominal compliances are always the same therefore it is impossible to get a robust design with nominal compliance increasing.

In this paper, it will be shown that all of the compliance oriented approaches which are based on a single or combined statistical measure can be replaced by a compliance-function-shape-oriented robust approach in which the allowed-compliance-increment will be an explicitly defined design variable and for a given increment value the robust solution will be the theoretically best one.

Using the terminology of the classical variational problems, the proposed total compliance variation oriented approach [21] can be classified as a curve-length, surface-area, volume or generalized volume minimizing problem with a problem specific condition which constrains the maximum allowable increase of the nominal-compliance, expressed as a percentage of the original (deterministic) nominal compliance, the searching domain is defined implicitly as integration limits in the objective function formulation, and the usual equality relation is used to prescribe the allowable material volume expressed as a percentage of the total material volume.

The paper is organized as follows. Section 2 focuses on the problem-specific mathematical formulation and the numerical algorithm of the total-compliance-variation-oriented approach. The example used to illustrate the viability and efficiency of the proposed approach is presented in Section 3. Finally, some concluding remarks are presented in Section 4.

\section{Models and algorithms}

In this paper, for sake of simplicity but without loss of generality the theoretical model of the compliance-function-shape-oriented robust approach will be formulated only for symmetric 2D structures with one point load with uncertain direction and magnitude.
In this case, using the terminology of the classical variational problem, the total-compliance-variation oriented approach will be a surface-area-minimization problem with a maximally allowed compliance increment constraint. Without any mathematical proof, it simply follows from its definition that the total-compliance-variation is the theoretically best measure of robustness and its minimization model is the only one which has an explicitly defined and from engineering point of view an easily interpretable design parameter to control the robustness. It has to be emphasized that in the combined expected compliance and compliance variation or standard deviation oriented measures the best weighting schema searching process the weight factor is an artificial design parameter without any engineering meaning. In the case of combined compliance expectancy and variation measure, the problem will be more complicated because an additional normalizing factor has to be introduced in order to resolve the disparity problem between the different measure units.

The load with uncertain direction and magnitude can be defined by a two-dimensional vector:

$$
\boldsymbol{f}(m, d)=[m \cos (d), m \sin (d)],
$$

where the magnitude is denoted by $m$ and the direction by $d$

The volume-constrained total compliance variation (surface area) minimization model can be described in the following form:

$$
\begin{aligned}
& \boldsymbol{t v}(\boldsymbol{x}) \rightarrow \min , \\
& \boldsymbol{m} \boldsymbol{v}(\boldsymbol{x})=\varphi \bar{v}, \\
& \boldsymbol{K} \boldsymbol{U}=\boldsymbol{F}, \\
& \boldsymbol{c}(\dot{m}, \dot{d})=\tau \underline{c}, \\
& 0 \leq \boldsymbol{x} \leq 1,
\end{aligned}
$$

where $\boldsymbol{x}$ is the vector of design variables (the element densities), $\boldsymbol{t} \boldsymbol{v}(\boldsymbol{x})$ is the total-variation (surface-area) of the compliance function on the set of the feasible loads, $\boldsymbol{U}$ and $\boldsymbol{F}$ are the displacement and load vectors, respectively, $\boldsymbol{K}$ is the global stiffness matrix, $\boldsymbol{m v}(\boldsymbol{x})$ and $\bar{v}$ are the material volume and design domain volume, respectively, $\varphi$ is the prescribed volume fraction, $\boldsymbol{c}(m, d)$ is the nominal compliance function, $\underline{c}$ is the nominal compliance minimal compliance, and $\tau>1$ is the allowed maximum nominal compliance increase factor.

Exploiting the fact that load vector $\boldsymbol{F}$ has maximum two nonzero entries the surface-area of compliance function $\boldsymbol{t} \boldsymbol{v}(\boldsymbol{x})$ can be described as follows (see, e.g., Olver [22]): 
$\boldsymbol{t} \boldsymbol{v}(\boldsymbol{x})=\int_{d-\bar{d} \dot{m}-\bar{m}}^{d+\bar{d}} \int^{\dot{m}+\bar{m}} \sqrt{1+\left(\frac{\partial \boldsymbol{c}(m, d)}{\partial m}\right)^{2}+\left(\frac{\partial \boldsymbol{c}(m, d)}{\partial d}\right)^{2}} \boldsymbol{d} m \boldsymbol{d} d$

where

$$
\boldsymbol{c}(m, d)=\boldsymbol{f}(m, d) \boldsymbol{Q} \boldsymbol{f}(m, d)^{t}
$$

is the directional compliance function and $\boldsymbol{Q}$ is a $2 \times 2$ symmetric matrix consisting of such entries of $\mathbf{K}^{-1}$ which are needed for the compliance computation. It is worth to note, that the selected inverse elements can be computed without inverting matrix $\mathbf{K}$ as a whole.

The nominal (deterministic) load magnitude and direction are denoted by $\dot{m}$ and $\dot{d}$, the variations are defined by the $\dot{m}-\bar{m} \leq m \leq \dot{m}+\bar{m}$ and $\dot{d}-\bar{d} \leq d \leq \dot{d}+\bar{d}$ relations.

In the case of $2 \mathrm{D}$ topology optimization problems, the design domain is assumed to be rectangular and discretized with $n=e^{x} \times e^{y}$ square elements discretized with four nodes per element and two degrees of freedoms (DOFs) per node. Both nodes and elements are numbered columnwise from left to right.

The algorithm of the new robust approach has been developed in Matlab language as a variant of a very efficient 88 line Matlab code developed in [23] for the traditional deterministic SIMP-type volume-constrained compliance-minimization problem, starting from the famous 99 line code which was originally developed in [24]. To solve the large and nonlinear optimization problem the fmincon solver from the Matlab environment was used with semi-analytical objective and gradient function values. It is an open and very challenging question that what would be the most efficient numerical algorithm which could solve the problem within a more reasonable time.

In the expected compliance model, the standard deviation model, and their weighted combination the objectives and gradients can be generated symbolically using appropriate symbolic manipulation software. In this study, for all symbolic computation the Wolfram Mathematica package was used. These models differ from the total compliance variation model only by the objective function; therefore their description will be omitted here. In the following, the objective function of these models will be denoted by $\boldsymbol{e c}(\boldsymbol{x}), \boldsymbol{d c}(\boldsymbol{x})$, and $\boldsymbol{e d}(\boldsymbol{x})$, respectively, where $\boldsymbol{e d}(\boldsymbol{x})=\lambda \boldsymbol{e c}(\boldsymbol{x})+(1-\lambda) \boldsymbol{d c}(\boldsymbol{x})$ with an explicitly defined design parameter (weighting factor) .

Using the applied notations, the mathematical formulation of the traditional deterministic volume-constrained compliance minimization problem can be described as follows:

$$
\begin{aligned}
& \boldsymbol{n c}(\boldsymbol{x})=\boldsymbol{f}(\dot{m}, \dot{d}) \boldsymbol{Q f}(\dot{m}, \dot{d})^{t} \rightarrow \min , \\
& \boldsymbol{m v}(\boldsymbol{x})=\varphi \bar{v}
\end{aligned}
$$

$\boldsymbol{K} U=\boldsymbol{F}$,

$0 \leq \boldsymbol{x} \leq 1$

Therefore, using the optimal $\boldsymbol{Q}$ matrix the nominal minimal compliance will be defined in the following form:

$\underline{c}=\boldsymbol{f}(\dot{m}, \dot{d}) \boldsymbol{Q} \boldsymbol{f}(\dot{m}, \dot{d})^{t}$.

\section{Example}

In this section a popular bridge design problem will be presented with uncertain loading magnitude and direction to demonstrate the efficiency and viability of the new robust solution searching approach. In the non-parametric shape oriented approach the loading magnitude and direction are treated as uncertain-but-bounded parameters. In the parametric (stochastic) approach, the well-known three-sigma-rule is applied in each case to get the corresponding stochastic parameters. The example with reproducible numerical results as a benchmark problem may be used for testing the quality of exact and heuristic solution procedures to be developed in the future for robust topology optimization.

The Young's modulus is $E_{0}=1$, the Poisson's ratio is $v=0.3$ and the fixed volume fraction is $\varphi=0.25$. The penalization power is $p=3$ and we applied density filtering with filter radius $r_{\min }=3$.

In the parametric case, symbolically generated analytical functions and gradients can be used. However, in the total compliance oriented approach only semi-analytical functions and gradients can be generated because the symbolic integration has to be replaced by numerical integration.

The example, shown in Fig. 1, is a symmetric bridge with a ground structure of $60 \mathrm{~mm} \times 30 \mathrm{~mm} \times 1 \mathrm{~mm}$. An external point load $\boldsymbol{f}$ acts in the middle position of the bridge where the nominal magnitude is $\dot{m}=6$ and the nominal direction is $\alpha=3 \pi / 2$. The uncertain loading magnitude is described by the $\dot{m}-\bar{m} \leq m \leq m+\bar{m}$ symmetric relation, where $\bar{m}=3$. The directional uncertainty of the point load is defined by the symmetric $\dot{d}-\bar{d} \leq d \leq \dot{d}+\bar{d}$ relation, where $\dot{d}=\pi / 4$.

In the stochastic approach, applying the three-sigma-rule to the uncertain-but-bounded direction and size parameters, the means are defined as $\mu(m)=\dot{m}=3$ and 


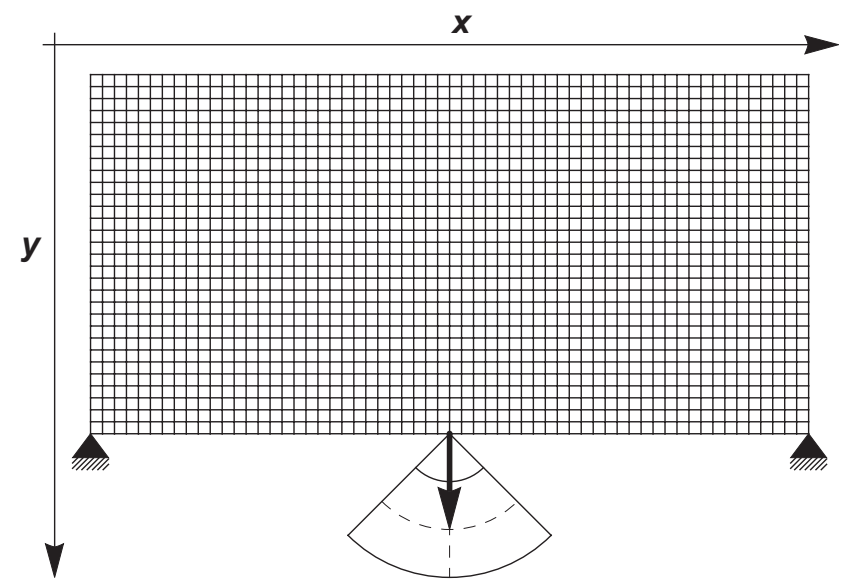

Fig. 1 The design domain, boundary conditions, and the applied point load with $3 \pi / 2+\pi / 4$ directional and $6 \pm 3$ magnitude uncertainty

$\mu(d)=\dot{d}=3 \pi / 2$, the standard deviations as $\sigma(m)=\bar{m} / 3$ and $\sigma(d)=\bar{d} / 3=\pi / 12$. As usual, it is assumed that loading magnitude $(m)$ and loading direction $(d)$ are normally distributed independent random variables (see Fig. 2).

The $\boldsymbol{n} \boldsymbol{c}$ minimal and the corresponding $\boldsymbol{t} \boldsymbol{v}$ minimal designs are presented in Figs. 3 and 4. The common plot of the compliance function shapes is shown in Fig. 5. It is very important to note that the hardly-detectable small differences in the design shapes can be able to cause relatively large differences in the compliance function shapes on the set of the feasible loads. In other words, an appropriate rearrangement of the available fixed amount of material may be able to smooth out the shape drastically without affecting the nominal-compliance-minimal compliance value.

The performance measures of the $\boldsymbol{n c}$ minimal and the corresponding $\boldsymbol{t} \boldsymbol{v}$ minimal solutions are presented in Table 1, in which each row describes an optimization process where the optimal objective function value is presented as a bold number in a light grey cell and the corresponding column label defines the currently used objective function.

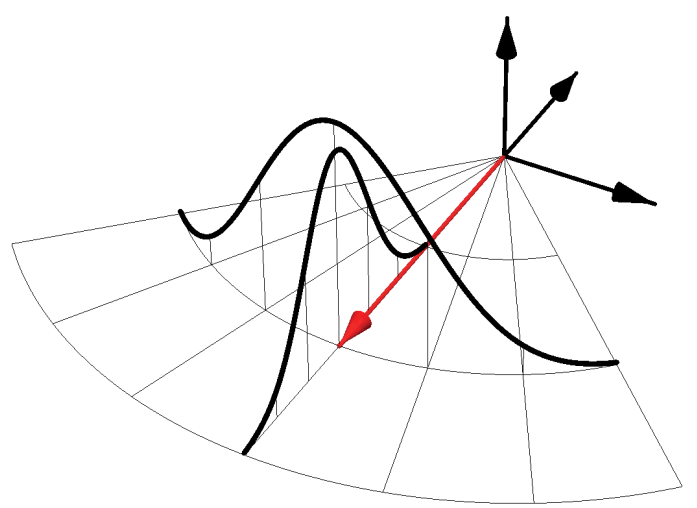

Fig. 2 Visualization of the multi-normal load with uncertain magnitude and direction

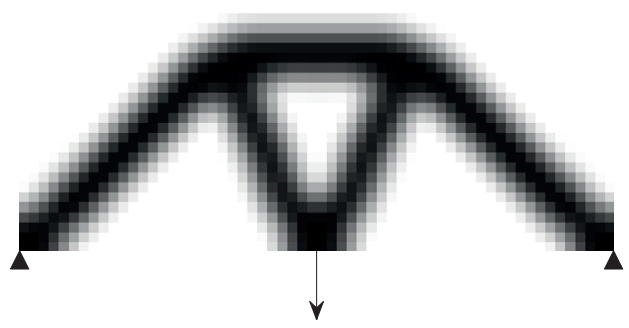

Fig. 3 The $\boldsymbol{n} \boldsymbol{c}$ minimal design

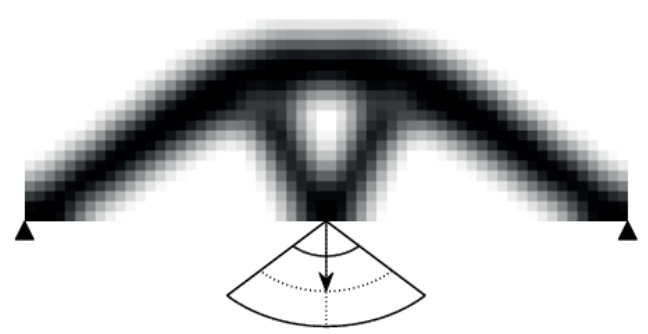

Fig. 4 The corresponding $\boldsymbol{t} \boldsymbol{v}$ minimal design with $\tau=1.00$

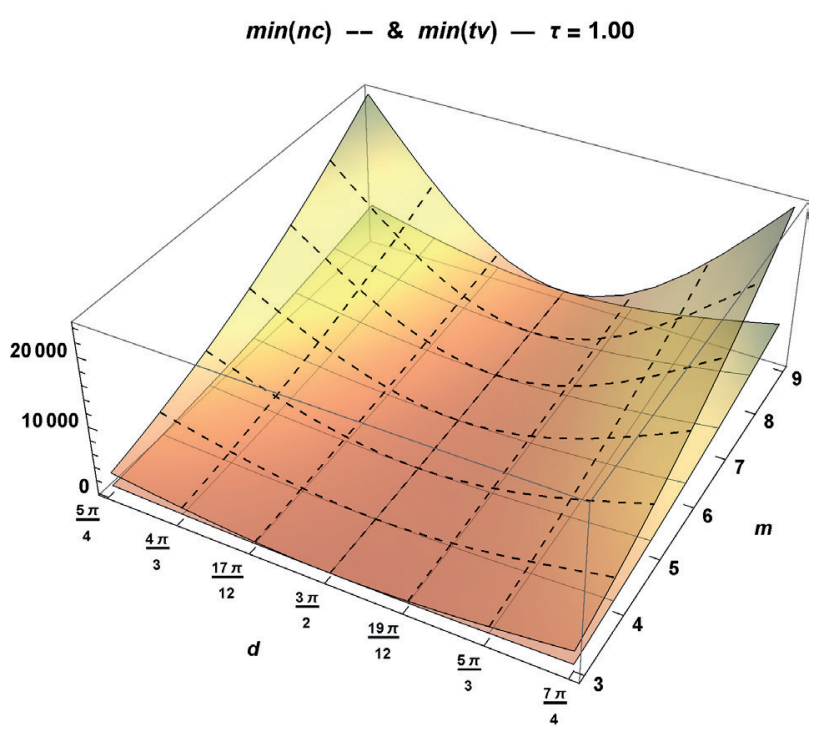

Fig. 5 The common plot of the compliance functions

Table 1 Performance measures of the $\boldsymbol{n c}$ and the corresponding $\boldsymbol{t} \boldsymbol{v}$ minimal solutions

\begin{tabular}{lccccc}
\hline$\tau$ & $\boldsymbol{n c}(\boldsymbol{x})$ & $\boldsymbol{t} \boldsymbol{v}(\boldsymbol{x})$ & $\overrightarrow{\boldsymbol{c}}(\boldsymbol{x})$ & $\overline{\boldsymbol{c}}(\boldsymbol{x})$ & $\overrightarrow{\boldsymbol{c}}(\boldsymbol{x})$ \\
\hline & $\mathbf{8 9 6 . 2}$ & 129641.3 & 24295.0 & 224.0 & 24070.9 \\
1.00 & 896.2 & $\mathbf{2 8 2 1 8 . 6}$ & 6792.9 & 224.0 & 6568.9 \\
\hline
\end{tabular}

The $\boldsymbol{e c}$ minimal and the corresponding $\boldsymbol{t} \boldsymbol{v}$ minimal designs are presented in Figs. 6 and 7. The common plot of the compliance function shapes is shown in Fig. 8. The performance measures of the $\boldsymbol{e c}$ and $\boldsymbol{t} \boldsymbol{v}$ minimal solutions are presented in Table 2.

The $\boldsymbol{d} \boldsymbol{c}$ minimal and the corresponding $\boldsymbol{t} \boldsymbol{v}$ minimal designs are presented in Figs. 9 and 10. The common plot of the compliance function shapes is shown in Fig. 11. The performance measures of the $\boldsymbol{d c}$ minimal and the corresponding $\boldsymbol{t} \boldsymbol{v}$ minimal solutions are presented in Table 3. 


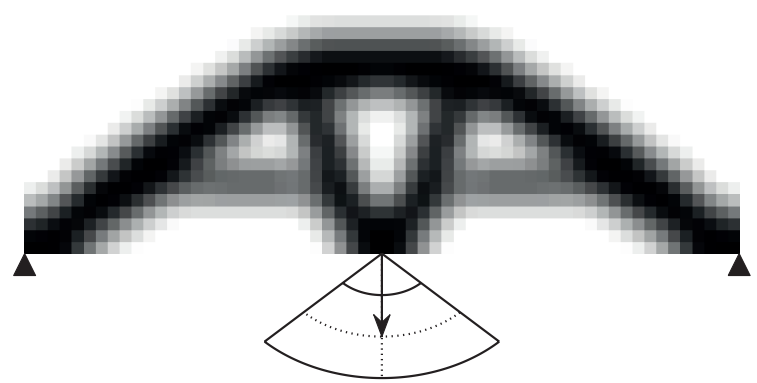

Fig. 6 The $e c$ minimal design

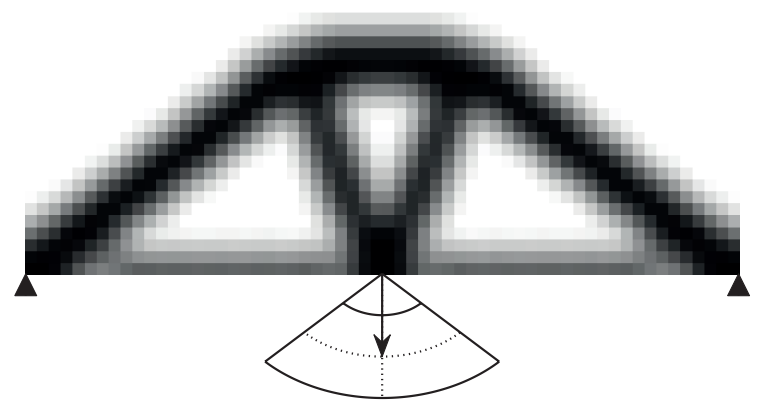

Fig. 7 The corresponding $\boldsymbol{t} v$ minimal design

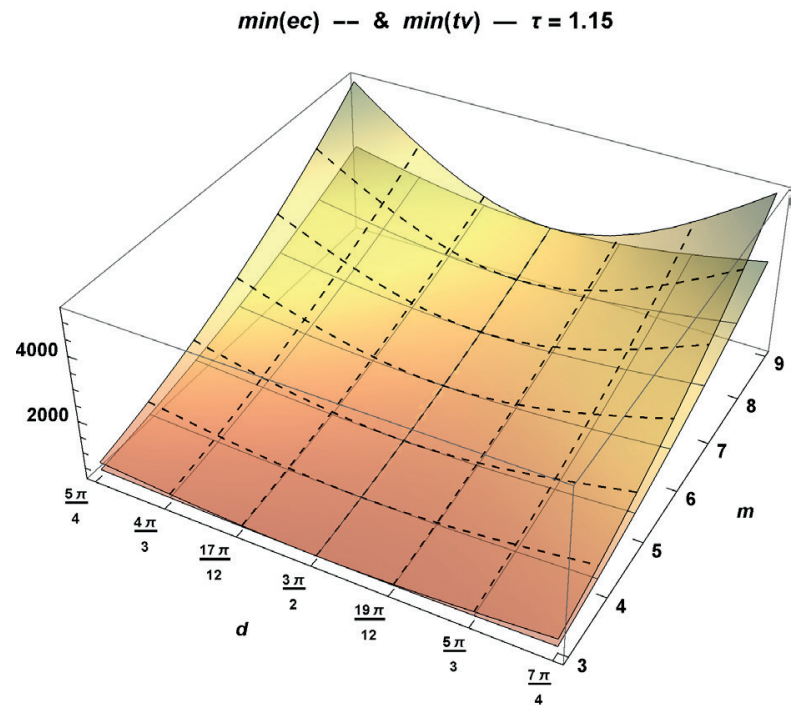

Fig. 8 The common plot of the compliance functions

Table 2 Performance measures of the $e c$ and the corresponding $t v$ minimal solutions

\begin{tabular}{lccccc}
\hline$\tau$ & $\boldsymbol{e c}(\boldsymbol{x})$ & $\boldsymbol{t} \boldsymbol{v}(\boldsymbol{x})$ & $\overrightarrow{\boldsymbol{c}}(\boldsymbol{x})$ & $\overline{\boldsymbol{c}}(\boldsymbol{x})$ & $\overrightarrow{\boldsymbol{c}}(\boldsymbol{x})$ \\
\hline & $\mathbf{1 0 2 6 . 8}$ & 5071.6 & 5328.3 & 256.7 & 5071.6 \\
1.15 & 1026.8 & $\mathbf{2 9 3 8 . 7}$ & 3195.4 & 256.7 & 2938.7 \\
\hline
\end{tabular}

The presented three examples well illustrate the fact that, according to the definition of the total compliance variation, its performance is at least as good as its original counterparts. It is also easily-understandable facts that, using the total variation measure, the higher the allowed nominal compliance increment the higher

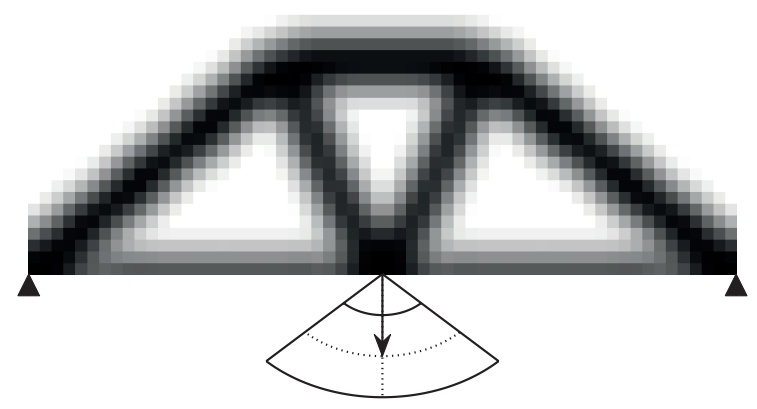

Fig. 9 The $\boldsymbol{d c}$ minimal design

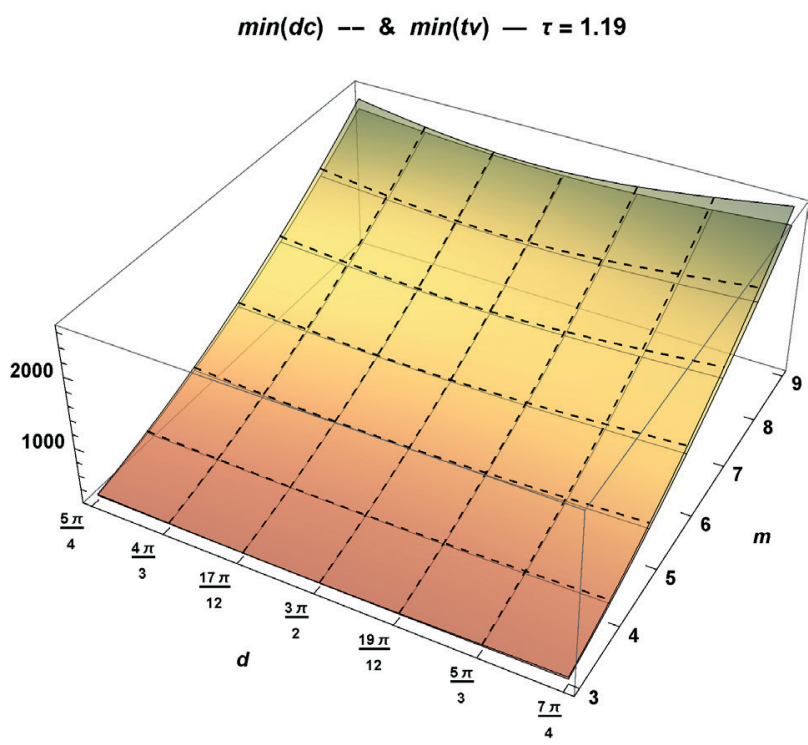

Fig. 10 The corresponding $t v$ minimal design

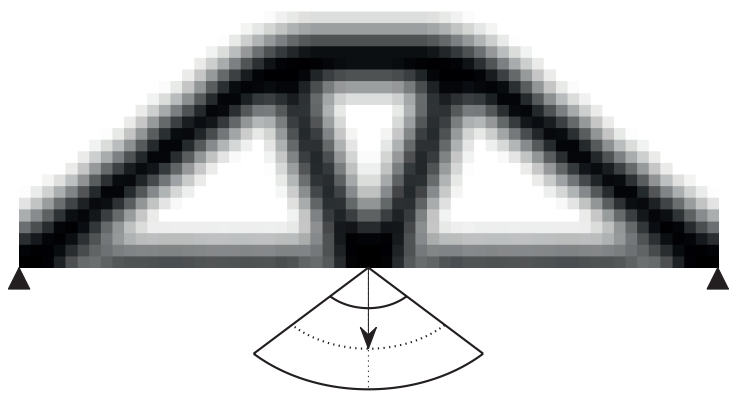

Fig. 11 The common plot of the compliance functions

Table 3 Performance measures of the $d c$ and the corresponding $t v$ minimal solutions

\begin{tabular}{lccccc}
\hline$\tau$ & $\boldsymbol{d} \boldsymbol{c}(\boldsymbol{x})$ & $\boldsymbol{t} \boldsymbol{v}(\boldsymbol{x})$ & $\overrightarrow{\boldsymbol{c}}(\boldsymbol{x})$ & $\overline{\boldsymbol{c}}(\boldsymbol{x})$ & $\overrightarrow{\boldsymbol{c}}(\boldsymbol{x})$ \\
\hline & $\mathbf{3 3 9 . 5}$ & 2394.8 & 2646.0 & 251.2 & 2394.8 \\
1.19 & 356.6 & $\mathbf{2 3 2 7 . 7}$ & 2595.0 & 267.3 & 2327.7 \\
\hline
\end{tabular}

the chance to get a more balanced compliance function shape. It is very interesting to see that performance of the standard deviation measure $(\boldsymbol{d c})$, as a single measure, is very close to the performance of the corresponding total variation ( $\boldsymbol{t} \boldsymbol{v})$ measure with $\tau=1.19$. If we select 
the $\tau \in[1.15,1.19]$ interval and define a combined measure as $\boldsymbol{e d}(\boldsymbol{x})=\lambda \boldsymbol{e c}(\boldsymbol{x})+(1-\lambda) \boldsymbol{d} \boldsymbol{c}(\boldsymbol{x})$ where $0 \leq \lambda \leq 1$ is an explicitly defined design parameter (weighting factor), we can generate all of the robust solutions in the $\boldsymbol{e c}(\boldsymbol{x}) \leq \boldsymbol{e d}(\boldsymbol{x}) \leq \boldsymbol{d c}(\boldsymbol{x})$ range. Only as an illustration, we present all of the results for $\lambda=0.5$. This case is enough to demonstrate, that from engineering point of view one of the most important performance parameters, namely the nominal compliance increment, remains hidden in the robust solution searching process. Because it is an implicit response variable, a try-and-error-like approach is needed to understand the hidden relation between the artificial objective function and the nominal compliance increment.

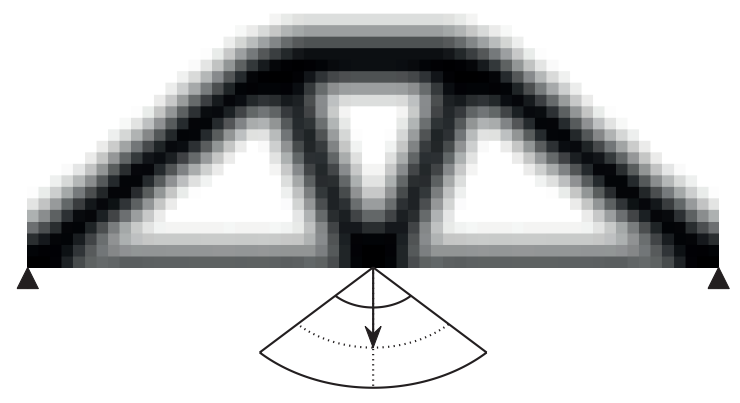

Fig. 12 The $\boldsymbol{e d}$ minimal design

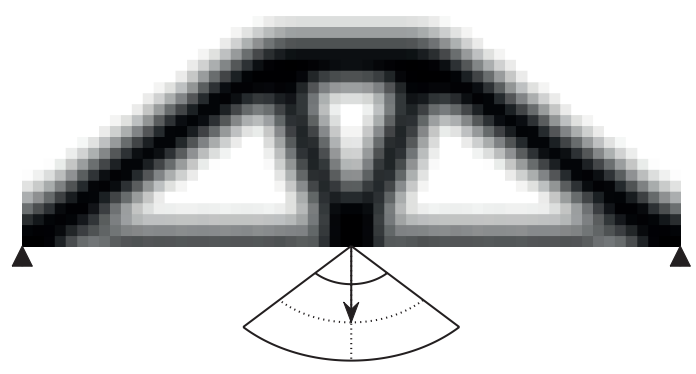

Fig. 13 The corresponding $\boldsymbol{t} v$ minimal design

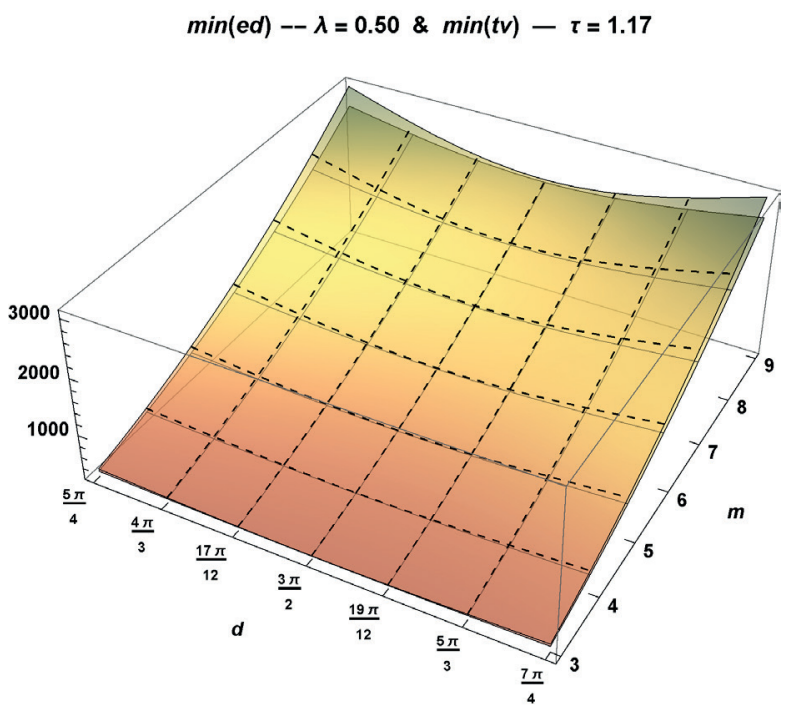

Fig. 14 The common plot of the compliance functions
Table 4 Performance measures of the $\boldsymbol{e d}$ and the corresponding $\boldsymbol{t} \boldsymbol{v}$ minimal solutions

\begin{tabular}{lccccc}
\hline$\tau$ & $\boldsymbol{e d}(\boldsymbol{x})$ & $\boldsymbol{t v}(\boldsymbol{x})$ & $\overrightarrow{\boldsymbol{c}}(\boldsymbol{x})$ & $\overline{\boldsymbol{c}}(\boldsymbol{x})$ & $\overrightarrow{\boldsymbol{c}}(\boldsymbol{x})$ \\
\hline & $\mathbf{7 3 7 . 3}$ & 5776.8 & 3085.0 & 261.8 & 2823.2 \\
1.17 & 722.9 & $\mathbf{4 2 6 8 . 2}$ & 2746.3 & 261.8 & 2484.5 \\
\hline
\end{tabular}

The $\boldsymbol{e d}$ and the corresponding $\boldsymbol{t} \boldsymbol{v}$ minimal designs are presented in Figs. 12 and 13. The common plot of the compliance function shapes is shown in Fig. 14. The performance measures of the $\boldsymbol{d c}$ and the corresponding $\boldsymbol{t} \boldsymbol{v}$ minimal solutions are presented in Table 4 .

In the following, corresponding to engineering way of thinking, we show that using the theoretically best $t v$ measure for example with design parameter (which means ten percentage nominal compliance increase) we get the required robust solution in exactly one step. The robust $\boldsymbol{t} v$ minimal design is presented in Fig. 15. The common plot of the $\boldsymbol{n} \boldsymbol{c}$ and $\boldsymbol{t} \boldsymbol{v}$ minimal compliance function shapes are shown in Fig. 16. The performance measures of the $\boldsymbol{n} \boldsymbol{c}$ and $\boldsymbol{t c}$ minimal solutions are shown in Table 5 where the $\boldsymbol{n c}$ minimal solution is only presented for the reason of easier comparison of the results.

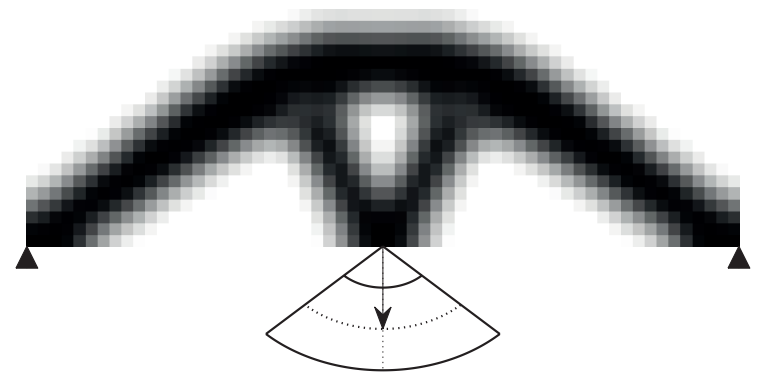

Fig. 15 The $\boldsymbol{t} \boldsymbol{v}$ minimal design with $\tau=1.10$

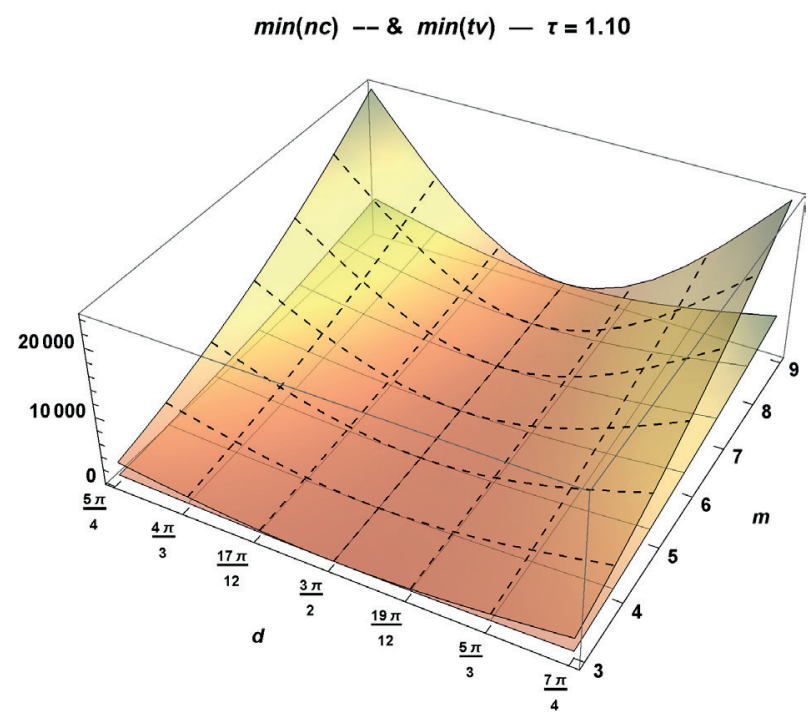

Fig. 16 The common plot of the compliance functions 
We have to note, that there is a practically never used parametric measure which performance is nearly the same as the performance of the corresponding total variation measure and this is the uniformly distributed expected compliance measure $(\boldsymbol{u c})$. The $\boldsymbol{u c}$ minimal and the corresponding $\boldsymbol{t} \boldsymbol{v}$ minimal designs are presented in Figs. 17 and 18. The common plot of the compliance function shapes is shown in Fig. 19.

The performance measures of the $\boldsymbol{u} \boldsymbol{c}$ and $\boldsymbol{t} \boldsymbol{v}$ minimal designs are presented in Table 6 .

In Fig. 20, we show common plot of the optimal $\boldsymbol{n c}$ and $t v$ compliance functions to visualize the fact that in the original $\boldsymbol{n} \boldsymbol{c}$ space the robust $\boldsymbol{t} \boldsymbol{v}$ minimal shape practically will be a plane.

\section{Conclusions}

In this paper, exploiting the fact that for each allowed compliance-increment value the total compliance variance is the theoretically best measure of robustness, it has been demonstrated that all of the parametric robustness measures can be replaced by the corresponding total compliance variance measure. In each case, the corresponding total compliance variance measure will be at least as good as

\begin{tabular}{cccccc}
\multicolumn{5}{c}{ Table $\mathbf{5}$ Performance measures of the $\boldsymbol{n} \boldsymbol{c}$ and the $\boldsymbol{t} \boldsymbol{v}$ minimal solutions } \\
\hline$\tau$ & $\boldsymbol{e d}(\boldsymbol{x})$ & $\boldsymbol{t} \boldsymbol{v}(\boldsymbol{x})$ & $\overrightarrow{\boldsymbol{c}}(\boldsymbol{x})$ & $\overline{\boldsymbol{c}}(\boldsymbol{x})$ & $\overrightarrow{\boldsymbol{c}}(\boldsymbol{x})$ \\
\hline & $\mathbf{8 9 6 . 2}$ & 129641.3 & 24295.0 & 224.0 & 24070.9 \\
1.10 & 985.8 & $\mathbf{2 3 2 7 . 7}$ & 4168.9 & 246.4 & 3922.4 \\
\hline
\end{tabular}

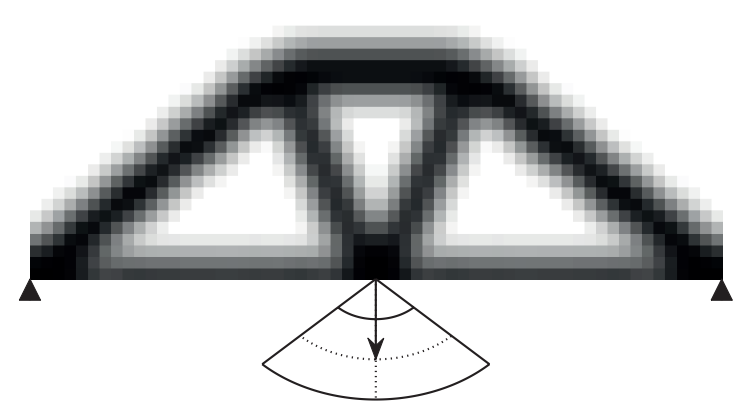

Fig. 17 The $\boldsymbol{u c}$ minimal design

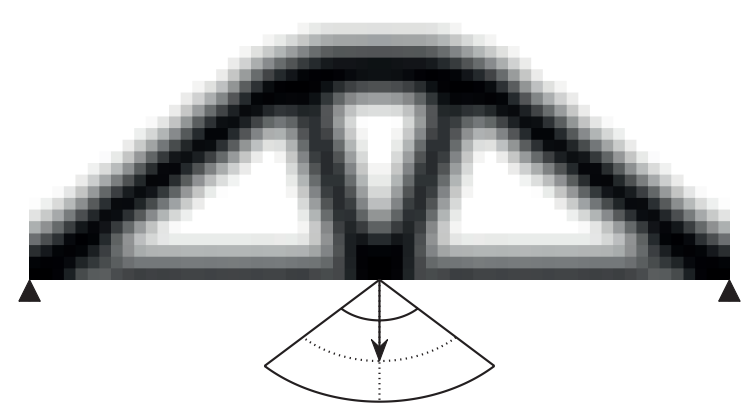

Fig. 18 The corresponding $t v$ minimal design

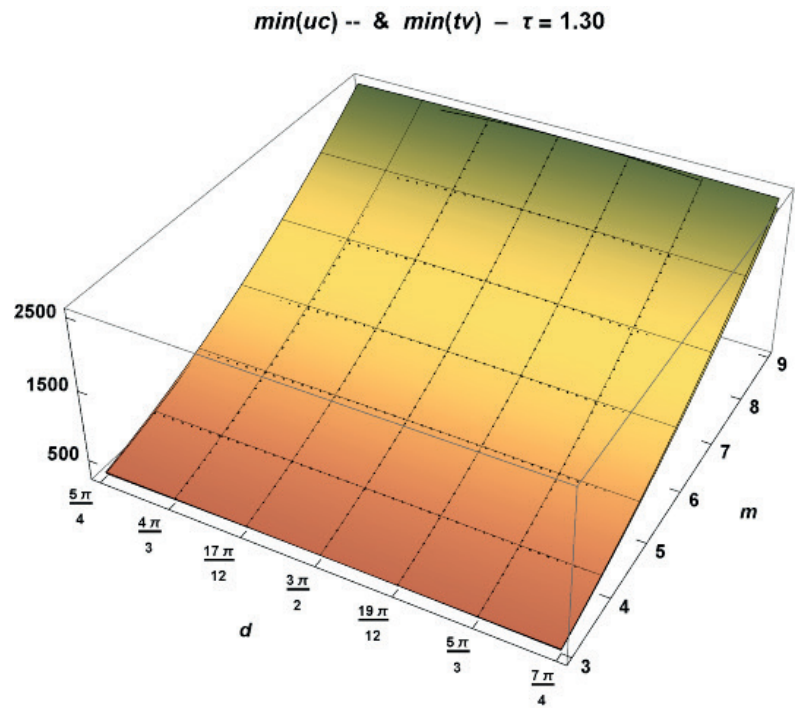

Fig. 19 The common plot of the compliance functions

Table 6 Performance measures of the $\boldsymbol{e d}$ and the corresponding $\boldsymbol{t} \boldsymbol{v}$ minimal solutions

\begin{tabular}{lccccc}
\hline$\tau$ & $\boldsymbol{e d}(\boldsymbol{x})$ & $\boldsymbol{t v}(\boldsymbol{x})$ & $\overrightarrow{\boldsymbol{c}}(\boldsymbol{x})$ & $\overline{\boldsymbol{c}}(\boldsymbol{x})$ & $\overrightarrow{\boldsymbol{c}}(\boldsymbol{x})$ \\
\hline \multirow{3}{*}{1203.8} & 3683.4 & 2564.9 & 265.2 & 2299.7 \\
\hline & & 3579.1 & 2575.3 & 284.8 & 2290.5 \\
\hline
\end{tabular}

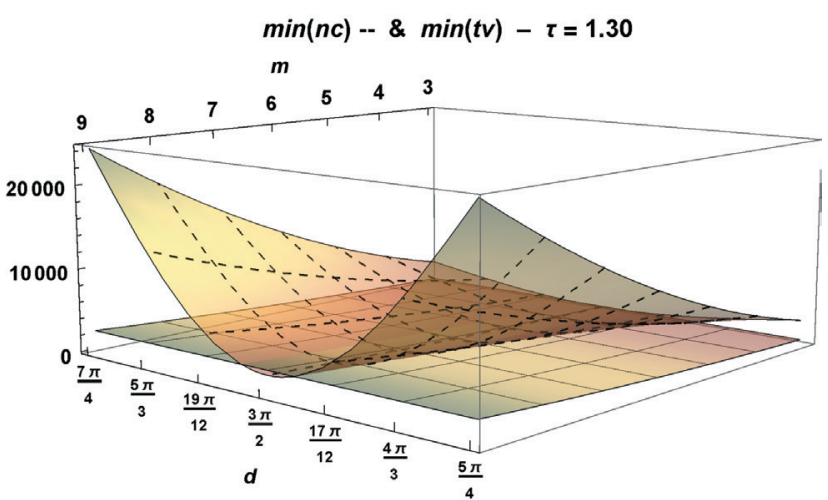

Fig. 20 The common plot of the $\boldsymbol{n c}$ and $\boldsymbol{t} \boldsymbol{v}$ compliance functions

any single or combined parametric measure independently from its statistical assumptions. From an engineering point of view, the most important feature of the proposed total compliance variance oriented robustness measure is that the best robust design searching process can be controlled by the maximally allowed nominal compliance increment which is an explicit and easy-to-understand design variable. A well-known and popular volume-constrained symmetric bridge problem with uncertain loading magnitude and direction has been used to illustrate the viability and efficiency of the proposed robust approach. The presented example as a benchmark problem can be used for testing the quality of exact and heuristic solution procedures to be developed in the future for the volume-constrained 
continuous robust topology optimization with uncertain loading magnitude and direction. A further application of the method could be to apply it to frame structures tasks examined by Lógó et al. [25-26] with limited residual strain energy capacity.

\section{References}

[1] Ben-Tal, A., Nemirovski, A. "Robust Truss Topology Design via Semidefinite Programming", SIAM Journal on Optimization, 7(4), pp. 991-1016, 1997.

https://doi.org/10.1137/S1052623495291951

[2] Lógó, J. "New Type of Optimality Criteria Method in Case of Probabilistic Loading Conditions", Mechanics Based Design of Structures and Machines, 35(2), pp. 147-162, 2007.

https://doi.org/10.1080/15397730701243066

[3] Schumacher, A., Olschinka, C. "Robust design considering highly nonlinear structural behavior", Structural and Multidisciplinary Optimization, 35, pp. 263-272, 2008.

https://doi.org/10.1007/s00158-007-0193-4

[4] Lógó, J., Ghaemi, M., Rad, M. M. "Optimal Topologies in Case of Probabilistic Loading: The Influence of Load Correlation", Mechanics Based Design of Structures and Machines, 37(3), pp. 327-348, 2009.

https://doi.org/10.1080/15397730902936328

[5] Lógó, J. "SIMP type topology optimization procedure considering uncertain load position", Periodica Polytechnica Civil Engineering, 56(2), pp. 213-219, 2012.

https://doi.org/10.3311/pp.ci.2012-2.07

[6] Pintér, E., Lengyel, A., Lógó, J. "Structural Topology Optimization with Stress Constraint Considering Loading Uncertainties", Periodica Polytechnica Civil Engineering, 59(4), pp. 559-565, 2015. https://doi.org/10.3311/PPci.8848

[7] da Silva, G. A., Cardoso, E. L., Beck, A. T. "Non-probabilistic robust continuum topology optimization with stress constraints", Structural and Multidisciplinary Optimization, 59, pp. 1181-1197, 2019. https://doi.org/10.1007/s00158-018-2122-0

[8] Wang, F., Jensen, J. S., Sigmund, O. "Robust topology optimization of photonic crystal waveguides with tailored dispersion properties", Journal of the Optical Society of America B, 28(3), pp. 387-397, 2011.

https://doi.org/10.1364/josab.28.000387

[9] Luo, Y., Zhou, M., Wang, M. Y., Deng, Z. "Reliability based topology optimization for continuum structures with local failure constraints", Computers and Structures, 143, pp. 73-84, 2014. https://doi.org/10.1016/j.compstruc.2014.07.009

[10] Liu, K., Paulino, G. H., Gardoni, P. "Reliability-based topology optimization using a new method for sensitivity approximation application to ground structures", Structural and Multidisciplinary Optimization, 54, pp. 553-571, 2016. https://doi.org/10.1007/s00158-016-1422-5

[11] Canelas, A., Carrasco, M., López, J. "A new method for reliability analysis and reliability-based design optimization", Structural and Multidisciplinary Optimization, 59, pp. 1655-1671, 2019. https://doi.org/10.1007/s00158-018-2151-8

\section{Acknowledgement}

The present study was supported by the Hungarian National Scientific and Research Foundation (OTKA) (grant K 119440).

[12] Chun, J., Song, J., Paulino, G. H. "System-reliability-based design and topology optimization of structures under constraints on first-passage probability", Structural Safety, 76, pp. 81-94, 2019. https://doi.org/10.1016/j.strusafe.2018.06.006

[13] Wang, L., Liang, J., Chen, W., Qiu, Z. "A nonprobabilistic reliability-based topology optimization method of compliant mechanisms with interval uncertainties", International Journal for Numerical Methods in Engineering, 119(13), pp. 1419-1438, 2019. https://doi.org/10.1002/nme.6097

[14] Torii, A. J. "Robust compliance-based topology optimization: A discussion on physical consistency", Computer Methods in Applied Mechanics and Engineering, 352, pp. 110-136, 2019. https://doi.org/10.1016/j.cma.2019.04.022

[15] Dunning, P. D., Kim, H. A., Mullineux, G. "Introducing Loading Uncertainty in Topology Optimization", AIAA Journal, 49(4), pp. 760-768, 2011. https://doi.org/10.2514/1.J050670

[16] Dunning, P. D., Kim, H. A. "Robust Topology Optimization: Minimization of Expected and Variance of Compliance", AIAA Journal, 51(11), pp. 2656-2664, 2013. https://doi.org/10.2514/1.J052183

[17] Zhao, J., Wang, C. "Robust topology optimization under loading uncertainty based on linear elastic theory and orthogonal diagonalization of symmetric matrices", Computer Methods in Applied Mechanics and Engineering, 273, pp. 204-218, 2014.

https://doi.org/10.1016/j.cma.2014.01.018

[18] Csébfalvi, A. "Robust Topology Optimization: A New Algorithm for Volume-constrained Expected Compliance Minimization with Probabilistic Loading Directions using Exact Analytical Objective and Gradient", Periodica Polytechnica Civil Engineering, 61(1), pp. 154-163, 2017. https://doi.org/10.3311/PPci.10214

[19] Peng, X., Wang, J., Li, J., Jiang, S., Bu, W., Yi, B., Zhou, S. "Robust Topology Optimization with Loading Magnitude and Direction Uncertainty", In: Advances in Mechanical Design, Proceedings of the 2017 International Conference on Mechanical Design (ICMD2017), Prague, Czech Republic, 2018, pp. 455-466. 2018. https://doi.org/10.1007/978-981-10-6553-8_31

[20] Csébfalvi, A., Lógó, J. "A critical analysis of expected-compliance model in volume-constrained robust topology optimization with normally distributed loading directions, using a minimax-compliance approach alternatively", Advances in Engineering Software, 120, pp. 107-115, 2018.

https://doi.org/10.1016/j.advengsoft.2018.02.003 
[21] Csébfalvi, A. "A New Compliance-function-shapeoriented Robust Approach for Volume-constrained Continuous Topology Optimization with Uncertain Loading Directions", Periodica Polytechnica Civil Engineering, 62(1), pp. 219-225, 2018. https://doi.org/10.3311/PPci.11398

[22] Olver, P. J. "Chapter 21: The Calculus of Variations", In: Applied Mathematics Lecture Notes, University of Minnesota, Minneapolis, MN, USA, 2012, pp. 1148-1168.

[23] Andreassen, E., Clausen, A., Schevenels, M., Lazarov, B. S., Sigmund, O. "Efficient topology optimization in MATLAB using 88 lines of code", Structural and Multidisciplinary Optimization, 43, pp. 1-16, 2011.

https://doi.org/10.1007/s00158-010-0594-7

[24] Sigmund, O. "A 99 line topology optimization code written in Matlab", Structural and Multidisciplinary Optimization, 21, pp. 120-127, 2001.

https://oi.org/10.1007/s001580050176
[25] Lógó, J., Movahedi Rad, M., Knabel, J., Tauzowski, P. "Reliability based design of frames with limited residual strain energy capacity", Periodica Polytechnica Civil Engineering, 55(1), pp. 13-20, 2011. https://doi.org/10.3311/pp.ci.2011-1.02

[26] Lógó, J., Rad, M. M., Tamássy, T., Knabel, J., Tauzowski, P. "Reliability Based Optimal Design of Frames with Limited Residual Strain Energy Capacity", In: Topping, B. H. V., Costa Neves, L. F., Barros, R. C. (eds.) Proceedings of the Twelfth International Conference on Civil, Structural and Environmental Engineering Computing, Civil-Comp Press, Stirlingshire, UK, 2009, Paper 52.

https://doi.org/10.4203/ccp.91.52 